\title{
Intervenções para cuidadores de sobreviventes de acidente vascular cerebral: revisão sistemática
}

\author{
Jaine Kareny da Silva, ${ }^{1}$ Karla Ferraz dos Anjos, ${ }^{2}$ Vanessa Cruz Santos, ${ }^{3}$ \\ Rita Narriman Silva de Oliveira Boery, ${ }^{1}$ Darci de Oliveira Santa Rosa ${ }^{2}$ \\ e Eduardo Nagib Boery ${ }^{1}$
}

Como citar Silva JK, Anjos KF, Santos VC, Boery RNSO, Santa Rosa DO, Boery EN. Intervenções para cuidadores de sobreviventes de acidente vascular cerebral: revisão sistemática. Rev Panam Salud Publica. 2018;v42:e114. https:/ / doi.org/10.26633/RPSP.2018.114

RESUMO Objetivo. Sintetizar e criticar a produção científica sobre as intervenções desenvolvidas para reverter a sobrecarga de cuidado e os fatores associados em cuidadores de sobreviventes de acidente vascular cerebral ( $A V C$ ).

Método. Revisão sistemática desenvolvida nas bases de dados PubMed e SciELO, Portal CAPES e Biblioteca Virtual em Saúde, utilizando as palavras-chave Stroke AND Caregivers AND Intervention $e$ Stroke AND Caregivers AND Clinical trial. Foram selecionados para a revisão oito artigos de ensaios clínicos randomizados (ECR) e controlados publicados no período de 2008 a 2017 nos idiomas inglês, português e espanhol.

Resultados. Os estudos foram desenvolvidos na Suécia, Taiwan, Alemanha, China, Índia e Estados Unidos. Intervenções psicoeducativas, apoio e aquisição de habilidades tiveram implicações positivas para cuidadores - nos âmbitos psicológico, físico e social, na qualidade dos cuidados e na aquisição dos conhecimentos dos cuidadores - e para sobreviventes - por exemplo, redução da utilização dos serviços de saúde e maior capacidade de autocuidado. A avaliação crítica dos artigos mostrou que nenhum atendeu todas as recomendações metodológicas para ECR. As principais limitações foram a incompletude dos dados e a heterogeneidade das intervenções.

Conclusão. As intervenções tiveram resultados positivos para cuidadores e sobreviventes. Contudo, estudos futuros devem considerar uma avaliação de longo prazo dos desfechos analisados, com descrição detalhada sobre o levantamento prévio das necessidades de cuidado que guiaram o estudo e disponibilização do material de apoio utilizado nas intervenções para permitir a reprodução da intervenção.

Palavras-chave Sobreviventes; acidente vascular cerebral; cuidadores; ensaio clínico.

\footnotetext{
Universidade Estadual do Sudoeste da Bahia, Programa de Pós-Graduação em Enfermagem e Saúde, Jequié (BA), Brasil. Correspondência: Jaine Kareny da Silva, jainekareny@yahoo.com.br

2 Universidade Federal da Bahia (UFBA), Programa de Pós-Graduação em Enfermagem, Salvador (BA), Brasil.

3 Universidade Federal da Bahia (UFBA), Instituto de Saúde Coletiva, Salvador (BA), Brasil
}

$\mathrm{O}$ acidente vascular cerebral (AVC) é uma síndrome neurológica que ocorre subitamente, sendo considerada a segunda causa de mortalidade global entre as doenças cardiovasculares, especialmente entre adultos e idosos (1). Na América Latina, o Brasil permanece como um dos países com maior risco de morte prematura por $\operatorname{AVC}(2,3)$.

Quando não é letal, o AVC causa incapacidade funcional e cognitiva em cerca de $45 \%$ dos sobreviventes, tornando-os dependentes de cuidados em domicílio após a alta hospitalar (4). Nesse contexto, 
emerge a figura do cuidador familiar, definido como uma pessoa com vínculos consanguíneos ou laços de afetividade, como amigos ou vizinhos (5).

Para além dos aspectos positivos advindos da função de cuidador, como sentimentos de felicidade e gratidão (6), vivenciar essa nova experiência acarreta sobrecarga que está relacionada a consequências negativas, especialmente sobre aspectos psicoemocionais, como estresse, ansiedade e depressão (7). Tais aspectos podem resultar, indiretamente, em prejuízos para a qualidade do cuidado prestado aos sobreviventes (8).

Em cuidadores, o estresse tem sido associado a ansiedade (9), piora da qualidade de vida (QV) (10), isolamento social (11) e risco de mortalidade (12); nos sobreviventes de AVC, por sua vez, tem sido associado a dificuldades na reabilitação e pior QV $(13,14)$. A depressão parece ser ainda mais grave para os cuidadores (15) e pode acarretar mudanças no comportamento e no humor dos sobreviventes de AVC (16). A literatura sugere que essas repercussões negativas, que ocorrem após a alta hospitalar do sobrevivente ao AVC, estão fortemente relacionadas ao déficit no apoio ou suporte social, sobretudo por parte dos profissionais de saúde, os quais não atendem adequadamente às necessidades de saúde dos cuidadores e nem os preparam para o cuidado em domicílio (7).

Visando a sanar essa carência assistencial, a última diretriz internacional do AVC reforça a urgência de ações para treinamento das famílias pelos órgãos públicos, de forma a auxiliar essas famílias no gerenciamento de mudanças no estilo de vida e comportamento de saúde (17). Contudo, essas recomendações nem sempre são prioridade. No Brasil, por exemplo, desde a criação do Guia Prático do Cuidador em 2008 (18), as políticas públicas de saúde voltadas para os cuidadores de sobreviventes de AVC não foram efetivas (19).

Estudos apontam a necessidade de planejar e de implementar intervenções que possam reverter essa sobrecarga dos cuidadores, com melhora na QV $(19,20)$, acesso a maior conhecimento sobre os cuidados e redução de sintomas emocionais como ansiedade e depressão (21). Entretanto, a melhor maneira para propiciar essa capacitação e suporte social ainda não é evidente.

Sendo assim, o objetivo do presente estudo foi sintetizar e criticar a produção científica sobre as intervenções desenvolvidas para reverter a sobrecarga de cuidado e os fatores associados em cuidadores de sobreviventes de AVC.

\section{MATERIAIS E MÉTODOS}

Foi realizada uma revisão sistemática a partir dos repositórios PubMed (https: / / www.ncbi.nlm.nih.gov/pubmed/), SciELO (http://www.scielo.org/php/ index.php), Portal CAPES (http://www. periodicos.capes.gov.br/) e Biblioteca Virtual em Saúde (BVS) (http://brasil. bvs.br/). Os artigos selecionados foram publicados no período de 2008 a junho de 2017. A busca dos artigos foi feita no período de 15 maio a 4 de junho de 2017 (última busca) por três examinadoras independentes. Por se tratar de uma revisão sistemática realizada exclusivamente a partir de ensaios clínicos randomizados (ECR) do tipo não farmacológico, o que a diferencia das revisões anteriores $(22,23)$, este levantamento fornece o mais elevado nível de recomendação terapêutica baseada em evidências científicas.

Primeiramente, a questão de investigação foi formulada a partir da definição de população, intervenção, controle e desfecho de interesse (estratégia PICO): qual o efeito de intervenções, em comparação ao tratamento padrão, para reverter a sobrecarga de cuidado e os fatores associados em cuidadores de sobreviventes ao AVC? Nesse contexto, o tratamento padrão refere-se ao tratamento ou atividade que ocorreu anteriormente às novas intervenções analisadas nos artigos.

Foram então identificadas as palavraschave e as estratégias de busca - Stroke AND Caregivers AND Intervention; e Stroke AND Caregivers AND clinical trial - e selecionados os repositórios para pesquisa. A partir daí, foram delimitados os critérios de inclusão e exclusão e realizada a busca dos artigos a partir das palavras-chave individuais. Os critérios de inclusão adotados foram: disponibilidade de texto integral de acesso livre, publicação nos idiomas inglês, português ou espanhol, ser ECR e tratar de intervenção com cuidadores de sobreviventes de AVC. Foram excluídos estudos-piloto, teses, livros e capítulos, resumos, editoriais, artigos não revisados por pares e estudos com métodos mistos, qualitativos e transversais. Os resultados foram examinados para leitura de título, resumo e método e, finalmente, foram selecionados oito artigos para análise, com leitura na íntegra (figura 1), conforme a metodologia PRISMA (24).

Com o propósito de alcançar maior confiabilidade de seleção dos artigos rastreados, cada uma das três examinadoras realizou levantamentos nos bancos de dados de forma independente. Em caso de dúvida, todas eram envolvidas na leitura, minimizando, assim, a possibilidade de exclusão de artigos relevantes. Para homogeneizar os dados da busca e, posteriormente, a análise dos artigos identificados, utilizou-se um roteiro contendo informações sobre palavra-chave, base de dados, artigo identificado e motivos de exclusão e seleção do estudo.

Para sistematizar a produção dos dados, utilizaram-se dois formulários elaborados pelas pesquisadoras. $\mathrm{O}$ primeiro apresenta informações sobre nome do autor, população, desfecho, instrumentos e resultados das intervenções. O segundo apresenta informações sobre tipo, formato, duração da intervenção, número de sessões e profissionais envolvidos.

\section{RESULTADOS}

Foram analisados oito artigos resultantes de ECR com foco em intervenções para cuidadores de sobreviventes de AVC. Os estudos foram desenvolvidos na Suécia (25), Taiwan (26), Alemanha (27), China (28), Índia (29) e Estados Unidos (30-32). Em relação aos dados apresentados nos artigos, não houve padronização quanto ao número de participantes, sendo apresentadas amostras com 36 e 37 até 136 e 131 pessoas, respectivamente, em grupos de intervenção e controle. Em geral, os cuidadores foram cônjuges e do sexo feminino, tanto jovens como idosos.

Os principais desfechos primários analisados nos cuidadores foram relacionados a aspectos emocionais, como a depressão (27,31-32), e mudança de vida $(31,32)$. Quanto aos sobreviventes, foram avaliados os desfechos secundários correspondentes ao autocuidado, utilização do serviço de saúde (26) e independência funcional $(25-26,31)$, com uso de diferentes instrumentos (tabela 1).

As intervenções foram do tipo apoio, aquisição de habilidade e atividades psicoeducativas, geralmente desenvolvidas por enfermeiros. As intervenções de apoio buscaram fornecer apoio emocional por meio de ajuda profissional ou interações com outros cuidadores que vivenciaram experiências similares. 
FIGURA 1. Estratégia de busca de artigos sobre intervenções para cuidadores de sobreviventes de AVC

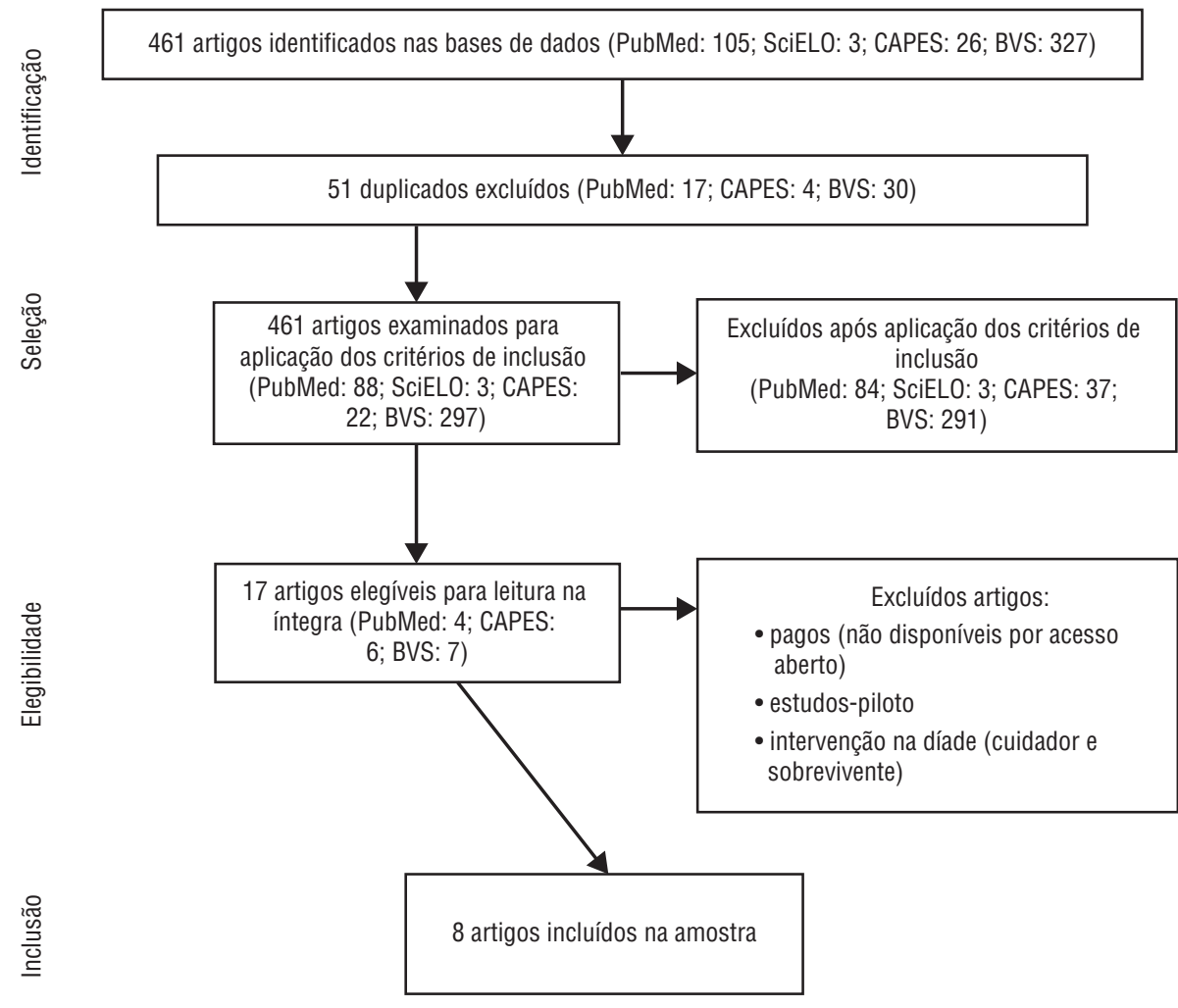

Nas intervenções de aquisição de habilidades, os cuidadores, em parceria com os profissionais, desenvolveram estratégias para a resolução de problemas, o gerenciamento do estresse e o estabelecimento de metas para a melhoria do cuidado. Por fim, as atividades psicoeducativas referiram-se a ações educativas que, por meio da orientação sobre a doença e o manejo do cuidado, promovem a melhoria da regulação das emoções dos cuidadores e, por conseguinte, aumentam o seu bem-estar (22).

As intervenções de apoio envolveram discussões nos formatos presencial, por telefone ou on-line, com participação de outros cuidadores ou individualmente com o profissional de saúde $(25,26$, 29 , 30). A intervenção para aquisição de habilidades envolveu estratégias para rastrear sintomas depressivos $(27,31,32)$ e resolver problemas emocionais e preocupações do cuidador (27).

As intervenções psicoeducativas abordaram informações sobre dados gerais do AVC (por exemplo, tipos, sinais e sintomas, prevenção e tratamento) $(25,32)$, serviços sociais e de saúde, gerenciamento de comportamento $(26,30,32)$ e cuidados com a saúde bucal do sobrevivente (28).
Esses foram os tipos mais utilizados de intervenções $(25,26,28$, 30, 32), geralmente combinadas a outras atividades, como apoio social. O uso das intervenções combinadas trouxe melhoria da sociabilidade e da qualidade dos cuidados (26), melhoria na saúde psicológica durante a intervenção e maior conhecimento sobre o AVC (25) em cuidadores e redução do uso de serviços de saúde pelos sobreviventes em longo prazo (26).

Foi relatada melhora nos sintomas depressivos com combinação de intervenções psicoeducativas e aquisição de habilidades após a finalização da intervenção (32). Entretanto, os resultados positivos do uso de estratégias de aquisição de habilidades isoladamente para a redução de danos psicológicos, como depressão, foram observados apenas durante o tratamento $(27,31)$. A melhora da depressão em cuidadores esteve associada ao aumento da independência funcional em sobreviventes ao AVC (31).

\section{Avaliação crítica dos estudos incluídos na revisão}

Na avaliação crítica, que foi baseada na recomendação PRISMA e na lista de verificação da declaração CONSORT (33), nenhum artigo atendeu $100 \%$ dos critérios sugeridos. Ainda, embora os oito artigos preenchessem a maioria dos critérios para um ECR do tipo não farmacológico, metade não tinha a informação do tipo de estudo no título $(25,27$, $28,32)$. Os dados sobre registro do estudo em banco de ensaios clínicos, como o ClinicalTrials.gov (32), protocolo do estudo (27) e fomento (apoio financeiro) (32) foram escassos.

Nos artigos analisados não houve padronização quanto à duração e ao número de sessões nas intervenções. Faltaram detalhes que permitissem a replicação, tais como: temas abordados $(26,29,30)$, material de apoio, tempo em cada sessão (28-31) e início da intervenção $(25,28)$. Um estudo publicou o material usado (27) e cinco descreveram que a intervenção mais comum no grupo controle foi o cuidado padrão $(26-28,31,32)$. Quanto ao cálculo do tamanho da amostra, definido pelo poder do estudo de $80 \%$ e nível de significância, três artigos não relataram esse item $(26,29,30)$.

No que se refere à geração de sequência da randomização, os principais tipos foram simples $(29)$, em blocos $(25,32)$ e estrato (27). Para a randomização foram utilizadas tabelas de números aleatórios, definidas por computador. A alocação foi realizada pelo pesquisador por meio de envelopes lacrados (25) e por centros externos à pesquisa $(27,32)$. A fase de implementação não foi descrita em seis artigos (25, 27-31). Os principais envolvidos no cegamento foram os participantes (26), coletadores e avaliadores $(27,28,31,32)$.

Houve incompletude nas informações sobre adesão dos profissionais ao protocolo da pesquisa (25-31), e quatro artigos descreveram a alocação por grupo $(27,28,31,32)$. Desses, dois relataram o tempo de experiência profissional $(27,28)$. As principais fontes de recrutamento dos cuidadores foram as clínicas de reabilitação $(27,30-32)$, unidades hospitalares $(25,26,32)$ e internação domiciliar (28). Entretanto, os critérios de inclusão e agrupamentos dos centros de saúde não foram informados.

Cinco artigos apresentaram o fluxograma com número de participantes e os motivos das perdas e exclusões (25-28, 32). Contudo, nenhum artigo descreveu a distribuição dos participantes e a alocação dos profissionais em cada centro de saúde (31). 
TABELA 1. Descrição dos principais achados dos estudos identificados na revisão sistemática sobre intervenções para cuidadores de sobreviventes de AVC

\begin{tabular}{|c|c|c|c|c|}
\hline Autor/ano (referência) & Populaçãoa & Desfecho & Instrumentos & Resultados ${ }^{a}$ \\
\hline Bakas et al., 2015 (32) & 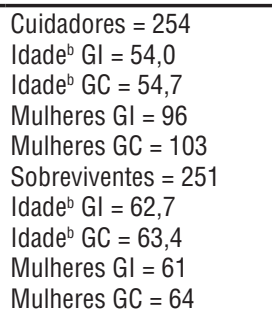 & $\begin{array}{l}\text { Cuidadores: } \\
\text { depressão, mudanças } \\
\text { de vida relacionadas } \\
\text { aos cuidados, dias } \\
\text { insalubres (número de } \\
\text { dias, em } 30 \text { dias, em } \\
\text { que a saúde física e/ou } \\
\text { mental do cuidador está } \\
\text { prejudicada) }\end{array}$ & $\begin{array}{l}\text { Cuidadores: Health Questionnaire } \\
\text { Depressive Symptom Scale; Bakas } \\
\text { Caregiving Outcomes Scale; dias } \\
\text { insalubres }\end{array}$ & $\begin{array}{l}\text { Cuidadores Gl: redução da depressão } \\
\text { em } 8,12,24 \text { e } 52 \text { semanas }(P<0,05) ; \\
\text { rotina de vida melhorou em } 12,24 \text { e } 52 \\
\text { semanas }(P<0,05) \text {; redução dos dias } \\
\text { insalubres em } 8 \text { semanas }(P<0,05) \text {. }\end{array}$ \\
\hline King et al., 2012 (31) & 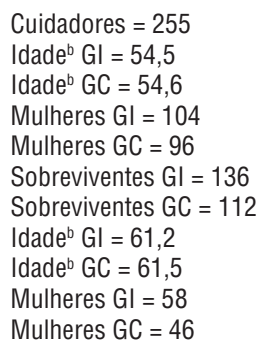 & $\begin{array}{l}\text { Cuidadores: } \\
\text { depressão, ansiedade, } \\
\text { capacitação, mudanças } \\
\text { de vida relacionadas } \\
\text { aos cuidados, } \\
\text { funcionamento familiar } \\
\text { Sobreviventes: MIFc }\end{array}$ & $\begin{array}{l}\text { Cuidadores: Center for Epidemiologic } \\
\text { Studies-Depression scale; Tension- } \\
\text { Anxiety 5-item subscale of the Profile of } \\
\text { Moods Scale short form; Preparedness } \\
\text { for Caregiving Scale; Bakas Caregiving } \\
\text { Outcomes Scale; Scale of the } \\
\text { McMaster Family Assessment Device } \\
\text { Sobreviventes: escore MIFc de } 18 \text { a } \\
96 \text { (GI/GC) }\end{array}$ & $\begin{array}{l}\text { Cuidadores Gl: redução da depressão } \\
\text { moderada e grave }(P<0,05) \text {; redução } \\
\text { da ansiedade }(P<0,05) \text {; capacitação: } \\
\text { SSE; mudança de vida }(P<0,05) \text {; } \\
\text { funcionamento familiar: SSE. } \\
\text { Sobreviventes: evolução clínica } \\
\text { inversamente proporcional à depressão } \\
\text { no cuidador }(P<0,05) \text {. }\end{array}$ \\
\hline Kuo et al., 2016 (28) & 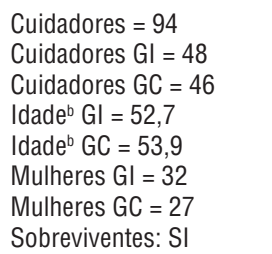 & $\begin{array}{l}\text { Cuidadores: } \\
\text { aprendizagem de } \\
\text { técnicas para cuidados } \\
\text { bucais domiciliários }\end{array}$ & $\begin{array}{l}\text { Cuidadores: Knowledge of Oral Care; } \\
\text { Behaviour of Oral Care; Family caregiver } \\
\text { Self-Efficacy of Oral Care; Attitude } \\
\text { towards Oral Care }\end{array}$ & $\begin{array}{l}\text { Cuidadores GI: melhorou } \\
\text { conhecimento sobre cuidados bucais } \\
(P<0,001) \text {; melhorou comportamento } \\
\text { bucal }(p<0,001) \text {; melhorou autoeficácia } \\
\text { para o cuidado bucal }(P<0,01) \text {; cuidado } \\
\text { oral da língua: SI. }\end{array}$ \\
\hline Malini, 2015 (29) & 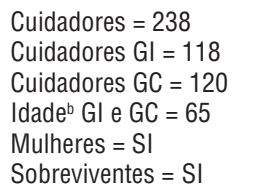 & $\begin{array}{l}\text { Cuidadores: forças do } \\
\text { sistema familiar (modo } \\
\text { de organização familiar) }\end{array}$ & $\begin{array}{l}\text { Cuidadores: Family System Strength } \\
\text { questionnaire }\end{array}$ & $\begin{array}{l}\text { Cuidadores: forças do sistema familiar: } \\
\text { aumento no Gl e estável no } \mathrm{GC}(P<0,05) \text {. }\end{array}$ \\
\hline Pfeiffer et al., 2014 (27) & 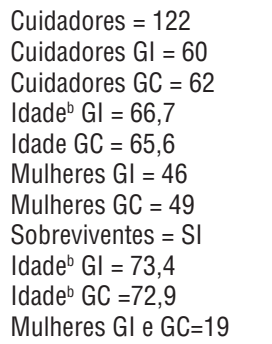 & $\begin{array}{l}\text { Cuidadores: } \\
\text { depressão, resolução } \\
\text { de problemas }\end{array}$ & $\begin{array}{l}\text { Cuidadores: Center for } \\
\text { Epidemiologic Studies -Depression } \\
\text { Scale; Sense of Competence } \\
\text { Questionnaire; Social Problem Solving } \\
\text { Inventory-Revised }\end{array}$ & $\begin{array}{l}\text { Cuidadores: redução da depressão no } \\
\text { GI após } 3(P<0,01) \text { e } 12 \text { meses }(P<0,05) \\
\text { satisfação da competência do cuidador } \\
\text { (GI e GC): SSE resolução de problema: } \\
\text { SSE. }\end{array}$ \\
\hline Shyu et al., 2010 (26) & 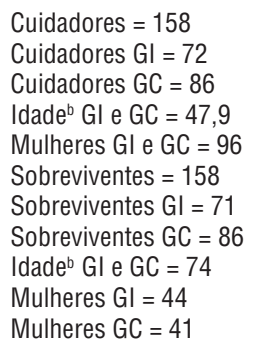 & $\begin{array}{l}\text { Cuidadores: } \\
\text { qualidade de vida; } \\
\text { qualidade dos cuidados } \\
\text { Sobreviventes: } \\
\text { capacidade de } \\
\text { autocuidado, utilização } \\
\text { do serviço }\end{array}$ & $\begin{array}{l}\text { Cuidadores: Medical Outcomes Study } \\
\text { Short Form; Family Caregiving } \\
\text { Consequence Inventory } \\
\text { Sobreviventes: Índice de Barthel } \\
\text { Chinês; questionário elaborado pelos } \\
\text { autores }\end{array}$ & $\begin{array}{l}\text { Cuidadores GI: qualidade de vida: } \\
\text { SSE; qualidade dos cuidados: } \\
\text { melhorou no } 6^{\circ} \text { mês }(P=0,00) \text {. } \\
\text { Sobreviventes: escore no Índice } \\
\text { de Barthel Chinês: SSE; reduziu a } \\
\text { institucionalização entre } 6^{\circ} \text { e } 12^{\circ} \text { mês } \\
\text { após a alta hospitalar. }\end{array}$ \\
\hline $\begin{array}{l}\text { Franzén-Dahlin et al., } \\
2008(25)\end{array}$ & 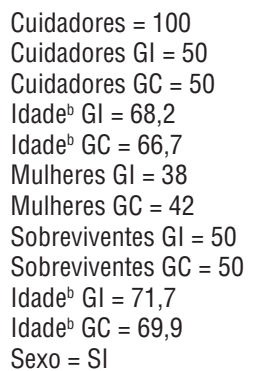 & $\begin{array}{l}\text { Cuidadores: saúde } \\
\text { psicológica }\end{array}$ & $\begin{array}{l}\text { Cuidadores: Comprehensive } \\
\text { Psychopathological Rating } \\
\text { Scale-Self-Affective } \\
\text { Sobreviventes: Barthel }\end{array}$ & $\begin{array}{l}\text { Cuidadores Gl: saúde psicológica } \\
\text { melhorou para aqueles com maior } \\
\text { participação em sessões }(P<0,05) \text {. }\end{array}$ \\
\hline
\end{tabular}


TABELA 1. (Continuação)

\begin{tabular}{|c|c|c|c|c|}
\hline Autor/ano (referência) & População $0^{a}$ & Desfecho & Instrumentos & Resultados $^{\mathrm{a}}$ \\
\hline Steiner et al., 2008 (30) & 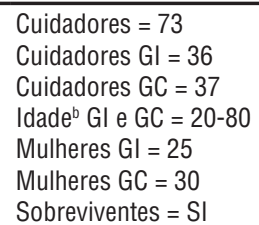 & $\begin{array}{l}\text { Cuidadores: apoio } \\
\text { emocional (familiares e } \\
\text { amigos), ajuda física } \\
\text { (familiares e amigos), } \\
\text { saúde }\end{array}$ & Cuidadores: Questionários próprios & $\begin{array}{l}\text { Cuidadores GI: relação positiva do } \\
\text { suporte emocional com ajuda física até } \\
3(P<0,01) \text { e } 12 \text { meses }(P<0,05) \text {, e com } \\
\text { saúde do cuidador aos } 6(P<0,05) \text { e } 12 \\
\text { meses }(P<0,01) \text {; correlação entre ajuda } \\
\text { física e saúde do cuidador: SSE; melhora } \\
\text { da saúde: SSE. }\end{array}$ \\
\hline
\end{tabular}

${ }^{a} \mathrm{GC}=$ grupo controle; $\mathrm{Gl}$ = grupo de intervenção; $\mathrm{SI}$ = sem informação; SSE = sem significância estatística.

${ }^{\mathrm{b}}$ Idade = média em anos.

${ }^{\mathrm{c}} \mathrm{MIF}=$ medida de independência funcional.

TABELA 2. Especificação do protocolo da intervenção dos estudos identificados na revisão sistemática sobre intervenções para cuidadores de sobreviventes de AVC

\begin{tabular}{|c|c|c|c|c|c|c|c|c|c|}
\hline \multirow[b]{2}{*}{ Autor/ano (referência) } & \multicolumn{3}{|c|}{ Tipo } & \multicolumn{4}{|c|}{ Formato } & \multirow[b]{2}{*}{ Tempo e número de sessões } & \multirow[b]{2}{*}{$\begin{array}{l}\text { Profissional } \\
\text { envolvido }\end{array}$} \\
\hline & $\begin{array}{l}\text { Aquisição de } \\
\text { habilidade }\end{array}$ & $\begin{array}{c}\text { Psico/ } \\
\text { educacional }\end{array}$ & Apoio & $\begin{array}{l}\text { Face- } \\
\text { a-face }\end{array}$ & Telefone & Internet & Correio & & \\
\hline Bakas et al., 2015 (32) & $\bar{x}$ & $\begin{array}{l}\text { Psico/ } \\
\text { educacional }\end{array}$ & - & $X^{a}$ & $\bar{x}$ & - & - & $\begin{array}{l}12 \text { semanas; } 9 \text { sessões telefônicas semanais } \\
\text { (8 semanas consecutivas e } 12^{\mathrm{a}} \text { semana) }\end{array}$ & Enfermeiro \\
\hline King et al., 2012 (31) & $\mathrm{X}$ & - & - & $x^{a}$ & $x$ & - & - & $\begin{array}{l}\text { Período: reabilitação aguda até } 3 \text { meses após } \\
\text { a alta hospitalar; } 10 \text { sessões telefônicas }\end{array}$ & $\begin{array}{l}\text { Enfermeiro; } \\
\text { estudante de } \\
\text { psicologia }\end{array}$ \\
\hline $\begin{array}{l}\text { Franzen-Dahlin et al., } \\
2008(25)\end{array}$ & - & Educacional & $x$ & $x$ & $x$ & - & - & $\begin{array}{l}7 \text { sessões/ } 7 \text { meses, ( } 6 \text { em grupo e } 1 \\
\text { telefonema) }\end{array}$ & $\begin{array}{l}\text { Enfermeiro; } \\
\text { médico }\end{array}$ \\
\hline Steiner et al., 2008 (30) & - & Educacional & $x$ & - & $x$ & $x$ & - & $\begin{array}{l}12 \text { meses; cuidador opta pelo horário de } \\
\text { atendimento }\end{array}$ & Enfermeiro \\
\hline Kuo et al., 2016 (28) & - & Educacional & - & $x$ & $x$ & - & - & $\begin{array}{l}2 \text { meses; } 2 \text { sessões mensais } \\
\text { (presencial e telefone) }\end{array}$ & Enfermeiro \\
\hline
\end{tabular}

a Encontro face-a-face para entrega de material educativo (folder).

As principais limitações dos estudos foram número elevado de perdas no seguimento e ausência de grupo controle de não cuidadores (25), ausência de coleta de dados basais (antes da intervenção) e intervenção em sobreviventes (26), possibilidade de os profissionais cegados terem acesso aos dados dos grupos $(27,28)$, aplicação de escala de tensão ao cuidador na presença do sobrevivente, possibilidade de o grupo controle ter recebido tratamento externo, viés de motivação (27), método estatístico não complexo nas análises (30), pouca adesão dos cuidadores em todas sessões, falta de reforço da intervenção (31) e restrição de idade (32).

\section{DISCUSSÃO}

Esta revisão sistemática reuniu diversas abordagens de intervenção implementadas com o intuito de reduzir a sobrecarga e os fatores associados em cuidadores de sobreviventes ao AVC nos diferentes continentes. Apesar do número escasso de intervenções voltadas para desfechos primários (contidos na tabela 1), com foco nos cuidadores, e da ausência de um consenso acerca dos desfechos analisados, existe preocupação internacional de pesquisadores em desenvolver ações que enfoquem a saúde dos cuidadores. O Brasil opõe-se a essa realidade, pois não foram encontrados estudos no período de busca.

Os aspectos culturais dos países e as características sociodemográficas dos cuidadores, como idade e sexo, sugerem relação entre si. Ser mulher cuidadora não diferiu entre os países onde as intervenções foram desenvolvidas. Essa característica cultural feminina prevalece em outros estudos $(34,35)$.

Infere-se ainda que os ensinamentos patriarcais arraigados no valor cultural familiar podem justificar as condições de escolha dos filhos como cuidadores. Por exemplo, na Ásia, a devoção aos pais é exacerbada; portanto, os filhos cuidam por obrigação tácita. Como resultado, as médias de idade entre cuidadores -47 e 52 anos $(26,28)$ - são menores do que aquelas relatadas nos outros seis estudos. No Brasil, os relatos indicam médias de idade semelhantes (57,1 e 47,41 anos) $(10,35)$. A explicação pode estar relacionada ao cumprimento da obrigação filial durante a velhice. Como reflexo, os cuidadores jovens podem sofrer maiores impactos, pois afastam-se de atividades acadêmicas, profissionais e sociais em um momento de vida produtiva, que também provoca prejuízos na contribuição ao setor previdenciário. Cuidadores com idade média $\geq 54$ anos possuem como aspecto negativo adicional o agravamento na saúde física e mental relacionado a comorbidades pré-existentes. 
Embora a idade e o sexo sejam variáveis importantes relacionadas à sobrecarga dos cuidadores, nota-se que a duração do papel de cuidador também resulta em sobrecarga, já que os cuidadores que prestaram assistência aos sobreviventes de AVC em períodos iguais ou superiores a 2 meses também apresentaram esse problema (35). Esses achados corroboram os de outros estudos $(19,36)$.

Apesar de a sobrecarga ser mensurada quanto aos aspectos financeiro, físico, social e emocional (37), a maioria dos estudos se preocupou em reverter dificuldades psicológicas. Para todos os estudos (25-32) da revisão, o fato de ser mulher esteve relacionado à maior ocorrência de sinais de depressão (38).

Perez et al. (39) afirmam que os cuidadores enfrentam fases semelhantes àquelas vivenciadas por seus familiares, dentre elas a depressão, que pode ser identificada desde a descoberta da doença crônica ou perpassar todo o período desde a reabilitação até a morte. Watanabe et al. (40) relatam que a assistência emocional aos cuidadores que exercem esse papel por longos períodos deve ser prestada continuamente.

Estudos sobre a QV dos cuidadores de doenças crônicas demonstraram que a saúde psicológica foi o domínio mais afetado $(19,36,41)$. Tais dados podem ter suscitado a elaboração de intervenções aos cuidadores de sobreviventes ao AVC com foco nas necessidades emocionais, pois o apoio social de profissionais de saúde é recomendado como fator atenuante da carga emocional (42).

Embora as intervenções tenham resultado em algum desfecho positivo para o cuidador, somente um estudo (32) conseguiu manter o efeito após a finalização da pesquisa. Considerando que o cuidado aos sobreviventes de AVC pode prolongar-se durante anos, esse dado tem reflexo na dimensão assistencial no que se refere ao apoio dos profissionais de saúde em período proporcional aos cuidados prestados (40) com a finalidade de reduzir a tensão do cuidador e melhorar a sua QV (43). Portanto, o apoio social formal deve ser intensificado em curto e longo prazo, por meio de orientações e capacitações de autocuidado e cuidado ao familiar enfermo (34), de modo a permitir o empoderamento desses cuidadores (44).

O enfermeiro foi o principal profissional envolvido nas intervenções. Suas notórias habilidades no âmbito da saúde coletiva na condução das ações de práticas educativas favorece o reconhecimento desse profissional como gerente de cuidado (45). Essa promoção da saúde passa pelo envolvimento nos princípios bioéticos da responsabilidade e da autonomia, os quais são vitais para a produção de resultados (46).

A aplicação das intervenções ocorreu sob dois formatos: presencial ou face a face (visita domiciliar) e não presencial (telefone e on-line). A unificação desses formatos tem sido frequente em outros estudos $(47,48)$, conforme preconizado pelas diretrizes da Organização Mundial do AVC, pois permite maior alcance dos resultados (17).

A estratégia presencial é uma intervenção efetiva, que fortalece o vínculo entre o profissional e os familiares; entretanto, pode ser onerosa e demandar tempo. Associá-la a recursos como telefone e Internet permite flexibilidade de horários, reduz o tempo de espera para o atendimento e evita desconforto decorrente do deslocamento (47), sendo essa associação um recurso válido para promover o autocuidado (49).

Apesar dos aspectos positivos, as intervenções devem ser cuidadosamente analisadas considerando os níveis de significância estatística, a diversidade dos resultados, as medidas usadas e os seus efeitos em curto e longo prazo. A declaração CONSORT foi desenvolvida inicialmente para auxiliar os autores em seus relatórios de maneira transparente e completa; contudo, também é útil para a avaliação dos relatos e a interpretação crítica. Em 2008, foi elaborada a extensão da CONSORT para avaliação dos ECR do tipo não farmacológico (33), com posterior atualização em 2017. Contudo, nessa revisão não utilizamos a versão atual, pois os artigos analisados foram anteriores à referida publicação. A recomendação PRISMA foi usada conjuntamente na crítica dos relatos.

O conceito de sobrecarga é multifacetado (37), sendo, portanto, necessária uma gama de intervenções para alcance dos desfechos. Em geral, as evidências mostram que intervir nos sintomas psicológicos é essencial para reduzir a sobrecarga do cuidador. Resultado semelhante foi relatado em outra revisão, ao identificar que intervenções psicossociais melhoram a saúde emocional e reduzem a sobrecarga, as dificuldades e as frustrações em cuidadores (23).

A confiabilidade dos resultados gerados pelos ECR é essencial para que outros pesquisadores possam basear-se nesses experimentos. Portanto, a exigência de registro dos ensaios tem aumentado por parte dos periódicos, pois permite maior visibilidade do processo de investigação e transparência (50), assim como a notificação das fontes de financiamento.

Apesar da diversidade no número de participantes, o cálculo amostral usado na maioria dos estudos demonstra seu poder de representatividade e significância estatística. Contudo, é necessário que a descrição também ocorra separadamente por grupos. Dessa maneira, a estimativa do efeito (por exemplo, risco relativo ou razão de chances) torna-se mais transparente.

Quanto aos três itens da randomização - sequência de geração, alocação e implementação -, notou-se a falta de transparência no relato em 50\% dos artigos. A descrição desses dados é necessária para apreensão da credibilidade do processo aleatório. O cegamento dos profissionais e participantes é particularmente difícil nos ECR, mas aplicar esse critério em coletadores e avaliadores, apesar de opcional, é importante para reduzir o viés de conduta dos profissionais (33). Entretanto, deve-se considerar que esse procedimento não é factível em certos casos.

A escassez de dados sobre experiência dos profissionais, critério de inclusão e complexidade dos centros de saúde onde as pesquisas foram realizadas ou os dados foram coletados pode afetar o efeito estimado da intervenção em análise (51). O fluxograma contendo as perdas de seguimento, com as respectivas justificativas, pode nortear futuras pesquisas quanto aos critérios de inclusão. Em estudos não farmacológicos, espera-se que os autores relatem as implicações terapêuticas no grupo controle e, posteriormente, reflitam sobre a intervenção, danos ou prejuízos e nível de experiência dos profissionais envolvidos, entre outros aspectos (51).

Este estudo tem por limitações as lacunas em termos de escopo, que incluem a falta de detalhes, a heterogeneidade dos desfechos, tipo e formato das intervenções e a incompletude de informações sociodemográficas, que pode interferir na validade externa dos resultados. O número de estudos identificados foi insuficiente para conduzir uma metanálise. Ademais, estudos recentes podem não ter sido incluídos nesta revisão devido ao 
tempo de atraso entre coleta de dados e a publicação de resultados. Apesar dessas limitações, esta revisão sintetiza os mais elevados níveis terapêuticos para melhorar os desfechos analisados, pois adotaram-se as recomendações PRISMA e a declaração CONSORT.

O emprego de cada tipo de intervenção (aquisição de habilidades, apoio e psicoeducação) dependerá dos desfechos (sobrecarga e fatores relacionados) a serem analisados. A combinação entre intervenções e entre diferentes formas de entrega (presencial ou não) pode potencializar o alcance dos resultados. Entretanto, deve-se atentar para a descrição minuciosa dos aspectos metodológicos e para a apresentação dos resultados. Recomendamos, portanto, maior detalhamento quanto a intervenções, desfechos analisados e acesso ao material utilizado nos estudos, pois esses dados poderão subsidiar a equipe de saúde, especialmente os enfermeiros, a implementar essas estratégias com maior confiabilidade e segurança nos serviços de saúde. Por conseguinte, os cuidadores poderão ser beneficiados com ações terapêuticas e preventivas em saúde, assim como os seus familiares que sobreviveram ao AVC.

Em conclusão, a heterogeneidade de instrumentos, desfechos e formato de entrega das intervenções para os cuidadores dificultou análise mais acurada dos estudos. Futuras pesquisas devem considerar uma avaliação de longo prazo dos desfechos analisados, com descrição detalhada sobre o levantamento prévio das necessidades dos cuidados que guiaram o estudo e disponibilização do material de apoio utilizado nas intervenções com vistas a promover a sua reprodutibilidade. Apesar de suas limitações, a presente revisão observou resultados positivos das intervenções para cuidadores de sobreviventes ao AVC, os quais podem guiar a construção de documentos e políticas públicas de saúde direcionadas a esses cuidadores.

Conflitos de interesse. Nada declarado pelos autores.

Declaração. As opiniões expressas no manuscrito são de responsabilidade exclusiva dos autores e não refletem necessariamente a opinião ou política da RPSP/PAJPH ou da Organização Pan-Americana da Saúde (OPAS).

\section{REFERÊNCIAS}

1. Wang H, Naghavi M, Allen C, Barber RM, Bhutta ZA, Carter A, et al. Global, regional, and national life expectancy, all-cause mortality, and cause-specific mortality for 249 causes of death, 1980-2015: a systematic analysis for the Global Burden of Disease Study 2015. Lancet. 2016;388(10053): 1459-544.

2. Ribeiro AL, Duncan BB, Brant LC, Lotufo PA, Mill JG, Barreto SM. Cardiovascular health in Brazil: trends and perspectives. Circulation. 2016;133(4):422-33.

3. Lotufo PA. Stroke is still a neglected disease in Brazil. Med J. 2015;133(6):457-9.

4. Benjamin EJ, Blaha MJ, Chiuve SE, Cushman M, Das SR, Deo R, et al. American Heart Association Statistics Committee and Stroke Statistics Subcommittee. Heart disease and stroke statistics-2017 update: a report from the American Heart Association. Circulation. 2017;135(10):e146-603.

5. Brasil, Ministério da Saúde, Secretaria de Atenção à Saúde, Departamento de Atenção Básica. Saúde mental. Brasília: Ministério da Saúde; 2013.

6. Couto AM, Castro EAB, Caldas AP. Vivências de ser cuidador familiar de idosos dependentes no ambiente domiciliar. Rev Rene. 2016;17(1):76-85.

7. Quinn K, Murray C, Malone C. Spousal experiences of coping with and adapting to caregiving for a partner who has a stroke: a meta-synthesis of qualitative research. Disabil Rehabil. 2014;36(3):185-98.

8. Mashau NS, Netshandama VO, Mudau MJ. Self-reported impact of caregiving on voluntary home-based caregivers in Mutale Municipality, South Africa. Afr J Prim Helth Care Fam Med. 2016;8(2):1-5.

9. Oosterveer DM, Mishre RR, van Oort A, Bodde K, Aerden LA. Anxiety and low life satisfaction associate with high caregiver strain early after stroke. J Rehabil Med. 2014;46(2):139-43.
10. Souza LR, Hanus JS, Libera LBD, Silva VM, Mangilli EM, Simões PV, et al. Sobrecarga no cuidado, estresse e impacto na qualidade de vida de cuidadores domiciliares assistidos na atenção básica. Cad Saude Coletiva. 2015;23(2):140-9.

11. Tooth L, McKenna K, Barnett A, Prescott C, Murphy S. Caregiver burden, time spent caring and health status in the first 12 months following stroke. Brain Inj. 2005;19(12):963-74.

12. Patterson TL, Grant I. Interventions for caregiving in dementia: physical outcomes. Curr Opin Psychiatry. 2003;16(6):639-33.

13. Han B, Haley WE. Family caregiving for patients with stroke: review and analysis. Stroke. 1999;30(7):1478-85.

14. Khalid W, Rozi S, Ali TS, Azam I, Mullen MT, Illyas S, et al. Quality of life after stroke in Pakistan. BMC Neurology. 2016;16(250): 1-12.

15. Olai L, Borgquist L, Svärdsudd K. Life situations and the care burden for stroke patients and their informal caregivers in a prospective cohort study. Ups J Med Sci. 2015;120(4):290-8

16. Gonzalez C, Bakas T. Factors associated with stroke survivor behaviors as identified by family caregivers. Rehabil Nurs. 2013;38(4):202-11.

17. Lindsay P, Furie KL, Davis SM, Donnan GA, Norrving B. World Stroke Organization global stroke services guidelines and action plan. Int J Stroke. 2014;9(Suppl A100): S4-13.

18. Brasil, Ministério da Saúde, Secretaria de Atenção à Saúde, Secretaria de Gestão do Trabalho e da Educação na Saúde. Guia prático do cuidador. (Série A. Normas e Manuais Técnicos.) Brasília: Ministério da Saúde; 2008. Disponível em: http:// bvsms.saude.gov.br/bvs/publicacoes / guia_pratico_cuidador.pdf Acessado em junho de 2018.
19. Costa TF, Costa KNFM, Fernandes MGM, Martins KP, Brito SS. Qualidade de vida de cuidadores de indivíduos com acidente vascular encefálico: associação com características e sobrecarga. Rev Esc Enferm USP. 2015;49(2):245-52.

20. Loureiro LSN, Fernandes MGM, Marques S, Nóbrega MML, Rodrigues RAP. Sobrecarga de cuidadores familiares de idosos: prevalência e associação com características do idoso e do cuidador. Rev Esc Enferm USP. 2013;7(5):1133-40.

21. Karahan AY, Kucuksen S, Yilmaz H, Salli A, Gungor T, Sahin M. Effects of rehabilitation services on anxiety, depression, care-giving burden and perceived social support of stroke caregivers. Acta Medica. 2014;57(2):68-72.

22. Bakas T, Clark PC, Kelly-Hayes M, King RB, Lutz BJ, Miller EL. Evidence for stroke family caregiver and dyad interventions: a statement for healthcare professionals from the American Heart Association and American Stroke Association. Stroke. 2014; 45(9):2836-52.

23. Greenwood N, Pelone F, Hassenkamp AM. General practice based psychosocial interventions for supporting carers of people with dementia or stroke: a systematic review. BMC Fam Pract. 2016;17(3):1-10.

24. Galvão TF, Pansani TSA, Harrad D. Principais itens para relatar revisões sistemáticas e meta-análises: a recomendação PRISMA. Epidemiol Serv Saude. 2015;24(2): 335-42.

25. Franzén-Dahlin Å, Larson J, Murray V, Wredling R, Billing E. A randomized controlled trial evaluating the effect of a support and education programme for spouses of people affected by stroke. Clin Rehabil. 2008;22(8):722-30.

26. Shyu, YIL, Kuo LM, Chen MC, Chen ST. A clinical trial of an individualised intervention programme for family caregivers 
of older stroke victims in Taiwan. J Clin Nurs. 2010;19:1675-85.

27. Pfeiffer K, Beische D, Hautzinger M, Berry JW, Wengert J, Hoffrichter R, et al. Telephone-Based Problem-Solving Intervention for Family Caregivers of Stroke Survivors: A Randomized Controlled Trial. J Consult Clin Psychol. 2014;82(4):628-43.

28. Kuo YW, Yen M, Fetzer S, Chiang LC, Shyu YIL, Lee TH, et al. A home-based training programme improves family caregivers' oral care practices with stroke survivors: a randomized controlled trial. Int J Dent Hygiene. 2016;14(2):82-91.

29. Malini MH. Impact of support group intervention on family system strengths of rural caregivers of stroke patients in India. Aust J Rural Health. 2015;23 (2):95-100

30. Steiner V, Pierce L, Drahuschak S, Nofziger E, Buchman D, Szirony T. Emotional support, physical help, and health of caregivers of stroke survivors. J Neurosci Nurs. 2008;40(1):48-54.

31. King RB, Hartke RJ, Houle T, Lee J, Herring G, Alexander-Peterson BS, et al. A problem-solving early intervention for stroke caregivers: one year follow-up. Rehabil Nurs. 2012;37(5):231-43.

32. Bakas T, Austin JK, Habermann B, Jessup $\mathrm{NM}$, McLennon SM, Mitchell PH, et al. Telephone assessment and skill-building kit for stroke caregivers: a randomized controlled clinical trial. Stroke. 2015;46: 3478-87.

33. Moher D, Hopewell S, Schulz KF, Montori V, Gotzsche PC, Devereaux PJ, et al. CONSORT 2010 explanation and elaboration: updated guidelines for reporting parallel group randomised trials. J Clin Epidemiol. 2010;63:e1-37.

34. Amendola F, Oliveira MAC, Alvarenga MRM. Influence of social support on the quality of life of family caregivers while caring for people with dependence. Rev Esc Enferm USP. 2011;45(4): 884-9.

35. Anjos KF, Boery RNSO, Pereira R, Pedreira LC, Vilela ABA, Santos VC, et al. Associação entre apoio social e qualidade de vida de cuidadores familiares de idosos dependentes. Cienc Saude Coletiva. 2015;20(5): 1321-30.

36. Silva IFG, Silva NCF, Gomes VAC, Dias BLM, Silva HMIL. Viver e cuidar após o acidente vascular cerebral. Rev Enf Ref. 2016;4(8):103-11.

37. Scazufca M. Brazilian version of the Burden Interview scale for the assessment of burden of care in carers of people with mental illnesses. Rev Bras Psiquiatr. 2002;24(1):12-7.

38. Cameron JI, Cheung AM, Streiner DL, Coyte PC, Stewart DE. Stroke survivor depressive symptoms are associated with family caregiver depression during the first 2 years poststroke. Stroke. 2011;42(2): 302-6.

39. Perez MP, Silva DPG, Couto TV. Percepções de um familiar de idoso hospitalizado na iminência de morte: um relato de caso. Arq Cienc Saude. 2009;16(1):34-9.

40. Watanabe A, Fukuda M, Suzuki M, Kawaguchi T, Habata T, Akutsu T, et al. Factors decreasing caregiver burden to allow patients with cerebrovascular disease to continue in long-term home care. J Stroke Cerebrovasc Dis. 2015;24(2):424-30.

41. Fhon JRS, Janampa JTG, Huaman TM, Marques S, Rodrigues RAP. Sobrecarga y calidad de vida del cuidador principal del adulto mayor. Av Enferm. 2016;34(3): 251-8.

42. Ross S, Morris RG. Psychological adjustment of spouses of aphasia stroke patients. Int J Rehabil Res. 1998;11(4):383-6.

43. Lino VTS, Rodrigues NCP, Camacho LAB, O'Dwyer G, Lima IS, Andrade MKN, et al. Prevalência de sobrecarga e respectivos fatores associados em cuidadores de idosos dependentes, em uma região pobre do Rio de Janeiro, Brasil. Cad Saude Publica. 2016;32(6):1-14.

44. Kruithof WJ, van Mierlo ML, Visser-Meily JM, van Heugten CM, Post MW. Associations between social support and stroke survivors' health-related quality of life: a systematic review. Patient Educ Couns. 2013;93(2):169-76.

45. Santos JLG, Pestana AL, Guerrero P, Meirelles BSH, Erdmann AL. Práticas de enfermeiros na gerência do cuidado em enfermagem e saúde: revisão integrativa. Rev Bras Enferm. 2013;66(2):257-63.

46. Cestari VRF, Florêncio RS, Moreira TMM, Pessoa VLMP, Barbosa IV, Lima FET, et al. Competências do enfermeiro na promoção da saúde de indivíduos com cardiopatias crônicas. Rev Bras Enferm. 2016;69(6): 1195-203.

47. Kim SS, Kim EJ, Cheon JY, Chung SK, Moon S, Moon KH. The effectiveness of home-based individual tele-care intervention for stroke caregivers in South Korea. Int J Nurs Rev. 2012;59(3): 369-75.

48. Pierce LL, Steiner V. Usage and design evaluation by family caregivers of a stroke intervention website. J Neurosci Nurs. 2013;45(5):254-61.

49. Rodrigues RCM, João TMS, Gallani MCBJ, Cornélio ME, Alexandre NMC. The "Moving Heart Program": an intervention to improve physical activity among patients with coronary heart disease. Rev Latinoam Enferm. 2013;21(spe):180-9.

50. García-Vello P, Smith E, Elias V, FlorezPinzon C, Revei L. Adherence to clinical trial registration in countries of Latin America and the Caribbean, 2015. Rev Panam Salud Publica. 2018;(42):1-9.

51. Martins J, Sousa LM, Oliveira AS. Recomendações do enunciado CONSORT para o relato de estudos clínicos controlados randomizados. Medicina. 2009;42(1): 9-21.

Manuscrito recebido em 29 de agosto de 2017. Aceito em versão revisada em 27 de abril de 2018 . 
ABSTRACT Objective. To synthesize and critically review the literature on interventions aimed at decreasing the burden of stroke survivor caregivers and other associated factors. Interventions for stroke
survivor caregivers:
a systematic review

Keywords
Method. The literature search for the present systematic review was performed in PubMed, SciELO, Coordination for the Improvement of Higher Education Personnel (CAPES) website, and the Virtual Health Library using the following search terms: Stroke AND Caregivers AND Intervention; and Stroke AND Caregivers AND clinical trial. Eight randomized controlled clinical trials (RCT) published between 2008 and 2017 were selected.

Results. The selected studies were performed in Sweden, Taiwan, Germany, China, India, and the United States. Psychoeducational, support, and skill acquisition interventions produced positive results for caregivers - in the psychological, physical, and social domains, in the quality of the care provided, and in the acquisition of knowledge by caregivers - and for stroke survivors - for example, decreased use of health care services and improved capacity for self-care. The critical evaluation of the studies showed that none met all the methodological requirements for RCTs. The main limitations were missing data and the heterogeneity of interventions.

Conclusion. The interventions had positive results for caregivers and survivors. However, future studies should consider long term assessments of the outcomes, with detailed description of the baseline needs that guided the study and the sharing of the support materials used to allow the intervention to be reproduced.

Survivors; stroke; caregivers; clinical trial.
RESUMEN

\section{Intervenciones para los cuidadores de los sobrevivientes de un accidente cerebrovascular: revisión sistemática}

Objetivo. Sintetizar y evaluar de manera crítica la producción científica sobre las intervenciones realizadas para revertir la sobrecarga del cuidado y los factores relacionados que afectan a los cuidadores de los sobrevivientes de un accidente cerebrovascular (ACV).

Método. Revisión sistemática efectuada en las bases de datos PubMed y SciELO, el Portal CAPES y la Biblioteca Virtual en Salud, con empleo de los grupos de palabras clave stroke AND caregivers AND intervention y stroke AND caregivers AND clinical trial. Se seleccionaron ocho estudios de ensayos clínicos aleatorizados y controlados que se publicaron del 2008 al 2017 en español, inglés y portugués.

Resultados. Los estudios se realizaron en Alemania, China, Estados Unidos, India, Suecia y Taiwán. Las intervenciones psicoeducativas, el apoyo y la adquisición de habilidades tuvieron repercusiones favorables para los cuidadores (en los aspectos psicológico, físico y social, en la calidad del cuidado y en la adquisición de conocimientos de los cuidadores) y para los sobrevivientes (por ejemplo, en el descenso de la utilización de los servicios de salud y el aumento de la capacidad de autocuidado). La evaluación crítica de los artículos mostró que en ninguno se tuvieron en cuenta todas las recomendaciones metodológicas para los ensayos clínicos aleatorizados. Las principales limitaciones fueron el carácter incompleto de los datos y la heterogeneidad de las intervenciones.

Conclusión. Las intervenciones tuvieron resultados positivos para los cuidadores y los sobrevivientes. Sin embargo, en estudios futuros se debe considerar la posibilidad de hacer una evaluación a largo plazo de los resultados analizados, con una descripción detallada de la determinación previa de las necesidades de cuidado que guiaron el estudio y de la disponibilidad del material de apoyo utilizado en las intervenciones para poder reproducirlas. 